\title{
Pruebas rápidas de anticuerpos contra el SARS-CoV-2: reporte de una experiencia en un Servicio de Emergencia
}

\author{
Rapid testing of SARS-CoV-2 antibodies: \\ Report of an experience in an Emergency Service
}

\author{
Jaime Antonio Collins-Camones', \\ Nikolai Grande-Castro², \\ Diana Jeanette Chávez-Montesinos ${ }^{3}$, \\ Sergio Enrique Alvizuri-Pastor ${ }^{3}$
}

\author{
Collins-Camones J, Grande-Castro N, Chávez-Montesinos D, Alvizuri- \\ Pastor S. Pruebas rápidas de anticuerpos contra el SARS-CoV-2: reporte \\ de una experiencia en un Servicio de Emergencia. Rev Soc Peru Med \\ Interna. 2020;33(3): 107-109. \\ https://doi.org/10.36393/spmi.v33i3.547
}

\begin{abstract}
RESUMEN
Objetivo: Determinar la positividad y los patrones de los resultados positivos de las pruebas rápidas de anticuerpos contra el SARS-CoV-2 en los pacientes atendidos en un Servicio de Emergencia. Materiales y métodos: Evaluación retrospectiva de las pruebas rápidas de anticuerpos contra el SARS-CoV-2 aplicadas durante el mes de junio/2020 en el Servicio de Emergencia del Hospital EsSalud G. Almenara, Lima, Perú. Resultados: Fueron evaluadas I 805 pruebas realizadas en igual número de pacientes. En 864 (48\%) de las pruebas fueron positivas, en el siguiente orden: patrón combinado de IgM-IgG en 635 (73\%), IgM en I34 (16\%) y, finalmente, de IgG en 95 (II\%).

Conclusiones: La positividad de las pruebas rápidas de anticuerpos contra el SARS-CoV-2 fue de $48 \%$, y el patrón combinado de IgM-IgG fue

el más frecuente, en tres cuartas partes de los casos confirmados.
\end{abstract}

Palabras clave: SARS-CoV-2, COVID-19, pruebas rápidas de anticuerpos, diagnóstico.

\section{ABSTRACT}

Objective: To determine the positivity and patterns of positive results of rapid antibody tests against SARS-CoV-2 in patitnts attending to an Emergency Service.

Material and methods: Retrospective evaluation of the rapid antibody tests against SARS-CoV-2 applied during the month of June 2020 in the Emergency Service of the Hospital EsSalud G. Almenara, Lima, Peru.

Results: We included 1805 tests performed on the same number of patients. In 864 (48\%) of the tests were positive, in

Médico internista e infectólogo. Servicio de Infectología, Departamento de Medicina Interna. Hospital EsSalud G. Almenara, Lima, Perú.

2 Médico patólogo clínico. Unidad de Inmuno-diagnóstico, Departamento de Patología Clínica. Hospital EsSalud G. Almenara, Lima, Perú.

3 Médico residente de medicina interna, Departamento de Medicina Interna. Hospital EsSalud G.Almenara, Lima, Perú.

4 Médico patólogo clínico. Departamento de Patología Clínica. Hospital EsSalud G.Almenara, Lima, Perú. the following order: combined IgM-lgG pattern in 635 (73\%), IgM in 134 (I6\%) and finally lgG in 95 (I I\%).

Conclusions: The positivity of the rapid antibody tests against SARS-CoV-2 was $48 \%$, and the combined pattern of $\lg M-\lg G$ was the most frequent in three quarters of the confirmed cases. Key words: SARS-CoV-2, COVID-19, rapid antibody test, diagnosis.

\section{INTRODUCCIÓN}

El Perú es uno de los países más afectados por el SARS$\mathrm{CoV}-2$ en el mundo. ${ }^{1}$ Las pruebas rápidas que detectan anticuerpos a partir de sangre periférica de pulpejo digital o venosa (en adelante, solo pruebas rápidas) para el SARSCoV-2 se están empleando como recurso diagnóstico de manera amplia en la población general. Tres cuartas partes de los diagnósticos de COVID-19 a nivel nacional se hicieron mediante este tipo de pruebas. ${ }^{2}$

Del mismo modo, las pruebas rápidas para el SARS-CoV-2 se están empleando para el diagnóstico en los servicios de emergencia de los hospitales. No obstante, hay escasa información disponible sobre su uso en este ámbito. Con 
base en ello, se buscó determinar la positividad de las pruebas rápidas para el SARS-CoV-2 empleadas en un servicio de emergencia, además de identificar cuáles fueron los patrones de los resultados positivos.

\section{MATERIAL Y MÉTODOS}

Se realizó una evaluación retrospectiva de las pruebas rápidas para el SARS-CoV-2 aplicadas durante el mes de junio del 2020 en la atención médica de todo el Servicio de Emergencia del Hospital EsSalud G. Almenara, de Lima ${ }^{1}$. Cabe señalar que solo se consideró una prueba por paciente, la primera. Los pacientes que pasan por el ambiente de triaje general de Emergencia y con sospecha de COVID-19 son derivados al ambiente de triaje respiratorio para la atención respectiva, y que termina con la decisión sobre si el manejo clínico será ambulatorio u hospitalario. Adicionalmente, los ambientes de hospitalización de la Emergencia están divididos en un área COVID-19 y en otra no COVID-19. Las pruebas fueron aplicadas tanto en pacientes de triaje respiratorio como en pacientes hospitalizados en las salas del Servicio de Emergencia o área COVID-19.

Las pruebas serológicas empleadas fueron: Covid-19 IgG/ IgM Combo Detection Kit-SD Biosensor; Cellex qSARSCoV-2 IgG/IgM Cassette Rapid Test Kit; y, Rapid Response Covid-19 IgG/IgM Test Cassette.

\section{RESULTADOS}

Fueron evaluadas 1805 pruebas rápidas para el SARSCoV-2, luego de excluir tres casos con resultado indeterminado. El 48\% (864/1805) de las pruebas fueron positivas, con los siguientes patrones de respuesta en orden de frecuencia: patrón combinado de inmunoglobulina IgMIg $\mathrm{G}$ en el $73 \%(635 / 864)$ de los casos; seguido por el de IgM con el 16\% (134/864); y, finalmente, el de IgG con el $11 \%(95 / 864)$. No hubo mayor variación de la positividad en triaje respiratorio respecto de la positividad general y de la misma en los otros sectores del Servicio de Emergencia, que incluye a los pacientes sin COVID-19 (Tabla 1). En cambio, sí existió una variación en las proporciones individuales de los anticuerpos IgM-IgG según el área de trabajo, consistente con el tipo de pacientes de las mismas (Tabla 2).

\section{DISCUSIÓN}

La positividad de las pruebas rápidas para el SARSCoV-2 del presente estudio representa poco más de la mitad de la sensibilidad alcanzable con estas pruebas.3,4 El hallazgo sugiere que las pruebas rápidas pueden ser más útiles en la atención de los pacientes que acuden a un Servicio de Emergencia si se utilizan, por un lado, en aquellos pacientes con adecuada sospecha de infección por SARS-CoV-2; y, por otro lado, si se toma en cuenta el tiempo que demanda la seroconversión de anticuerpos contra el SARS-CoV-2 en el curso de la enfermedad. Por su parte, la seroconversión con $\operatorname{IgM}$ e IgG va del $60 \%$ al $90 \%$ entre el sexto y el décimo segundo día de la enfermedad, respectivamente. Asimismo, el 100\% de la seroconversión puede alcanzarse en el décimo octavo día de la misma, es decir, a mediados de la tercera semana.5 Esto es importante porque una prueba rápida negativa en la segunda semana del curso clínico, en un paciente nuevo con alta sospecha de COVID-19, no descarta el diagnóstico y, en consecuencia, se puede proceder con una prueba molecular en la medida de lo posible, mientras se indican las medidas terapéuticas de acuerdo a la sospecha clínica. En otras palabras, no hay que descartar el diagnóstico de COVID-19 con base solo en la prueba rápida. De no contar con una prueba de diagnóstico molecular, cabe una nueva prueba rápida en el momento más oportuno. Esto no excluye de proceder con una tomografía de tórax de acuerdo a la magnitud o severidad del cuadro clínico, dada la alta sensibilidad y precocidad de sus hallazgos. 6

Por otra parte, los patrones de respuesta de anticuerpos hallados en el presente estudio son consistentes con lo descrito. ${ }^{7}$ Asimismo, la investigación propuesta es transversal, por lo que no es posible hacer un correlato estrecho entre los resultados individuales de anticuerpos con el curso clínico de la enfermedad, dado que la seroconversión con IgG puede preceder a la seroconversión con $\operatorname{IgM}$, como ha sido observado y caracterizado en un estudio longitudinal prospectivo, en discordancia al patrón de respuesta inmune habitual dentro del contexto de enfermedades infecciosas. ${ }^{5}$

La prueba de reacción en cadena de polimerasa con

\begin{tabular}{|c|c|c|c|}
\hline & Total & Triaje respiratorio (TR) & Fuera de TR \\
\hline & I 805 & 514 & | 291 \\
\hline Positividad (\%) & $864(48 \%)$ & $258(50 \%)$ & 606 (47\%) \\
\hline
\end{tabular}

Tabla 2. Patrones de respuesta según el área del Servicio de Emergencia. HNGA, junio del 2020.

$\begin{array}{llll}\text { Tipo de anticuerpo } & \text { Total (\%) } & \text { Triaje respiratorio (\%) } & \text { Fuera de Triaje Respiratorio (\%) } \\ & \mathbf{8 6 4 ( 1 0 0 )} & 258(100) & 606(100) \\ \text { IgM-lgG } & 635(73) & 185(72) & 450(74) \\ \lg M & 134(16) & 56(22) & 78(13) \\ \lg G & 95(11) & 17(6) & 78(13)\end{array}$


transcripción inversa o reversa (RT-PCR, por sus siglas en inglés) es la prueba estándar para el diagnóstico definitivo del SARS-CoV-2. No obstante, el requerimiento en el laboratorio de equipamiento especial y personal especializado, sumado al tiempo que gira alrededor de la prueba desde la toma de la muestra hasta disponer de los resultados, limitan su empleo a gran escala tanto en el ámbito clínico u hospitalario como en el de salud pública o comunitario. ${ }^{4,8}$ Las versiones rápidas de las pruebas moleculares para el SARS-CoV-2 aún están en desarrollo y evaluación. En contraste, las pruebas rápidas se han desarrollado en menos tiempo, lo cual ha permitido que se empleen como herramienta para el diagnóstico y control de la epidemia. No obstante, estas pruebas continúan en evaluación y desarrollo para mejorar la precisión del diagnóstico en diferentes escenarios, por lo que su uso debe acompañarse de un adecuado juicio clínico, con el complemento o no de una prueba molecular según su disponibilidad. ${ }^{8}$

Otra alternativa o complemento a las pruebas rápidas son las pruebas serológicas automatizadas que pueden ser procesadas a gran escala y cuentan con mayor precisión diagnóstica que las pruebas rápidas, aunque también están en evaluación y desarrollo. ${ }^{8,9}$ En ese sentido, dependerá de la demanda y la organización del centro en la respuesta frente a la COVID-19 elegir entre uno u otro método, o ambos.

Resulta importante señalar que el presente estudio tiene algunas limitaciones. En primer lugar, se trata de una investigación retrospectiva y transversal. En segundo lugar, durante el periodo de estudio se utilizaron tres marcas comerciales de pruebas rápidas en el hospital. No obstante, respecto a lo primero, se revisó con acuciosidad la población de estudio en orden alfabético para evitar duplicaciones de pacientes y, aparte de ello, fue claro el registro de las pruebas realizadas en triaje respiratorio. En relación con lo segundo, la cantidad de pruebas rápidas incluidas en el estudio es grande y la lectura de los resultados estuvo a cargo del personal especializado (tecnólogos médicos o médicos patólogos clínicos).

En conclusión, la positividad de las pruebas rápidas para el SARS-CoV-2 en el Servicio de Emergencia del Hospital EsSalud G. Almenara fue de 48\%, y el patrón combinado de IgM-Ig G fue el más frecuente, en tres cuartas partes de los casos confirmados.

\section{REFERENCIAS BIBLIOGRÁFICAS}

1. Johns Hopkins University \& Medicine. Coronavirus Resource Center, World Map. [Internet]. [Consulted July 11, 2020]. Available from: https://coronavirus. jhu.edu/map.html.

2. Ministerio de Salud, Perú. Sala situacional COVID-19 [Internet]. [Consultado 11 de julio de 2020]. URL disponible en: https:// covid19.minsa.gob.pe/sala situacional.asp.

3. Instituto Nacional de Salud, Perú. Precisión diagnóstica de pruebas rápidas de detección de anticuerpos para el SARS-CoV-2. Aramburú A. Lima: Unidad de Análisis y Generación de Evidencia en Salud Pública. INS. Marzo de 2020. Serie Revisiones Rápidas No 1 - 2020.

4. Castro R, Luz PM, Wakimoto MD, Veloso VG, Grinsztejn B, Perazzo H. COVID-19: meta-analysis of diagnostic test accuracy of commercial assays registered in Brazil. Braz J Infect Dis. 2020; 24(2): 180-7. doi: 10.1016/j.bjid.2020.04.003. Epub 2020 Apr18.

5. Long QX, Liu BZ, Deng HJ, Wu GC, Deng K, Chen Yk, et al. Antibody responses to SARS-CoV-2 in patients con COVID-19 [brief communications]. Nat Med. 2020 Jun; 26(6): 845-848. doi: 10.1038/s41591-020-0897-1. Epub 2020 Apr 29.

6. Ai T, Yang Z, Hou H, Zhan C, Chen C, Lv W, Tao Q, Sun Z, Xia L. Correlation of chest CT and RT-PCR testing in Coronavirus Diseases (COVID-19) in China: A report of 1014 cases. Radiology. 2020 Feb 26; 200642. doi: 10.1148/radiol.2020200642. Online ahead of print.

7. Li Z, Yi Y, Luo X, Xiong N, Liu Y, Li S, et al. Development and clinical application of a rapid IgM-IgG combined antibody test for SARS-CoV-2 infection diagnosis. J Med Virol. 2020 Feb 27; 10.1002/jmv.25727. doi: 10.1002/jmv.25727. Online ahead of print.

8. Younes N, AI-Sadeq DW, AI-Jighefee H, Younes S, AI-Jamal O, Daas H, Yassine HM, Nasrallah GK. Challenges in laboratory diagnosis of the novel coronavirus SARS-CoV-2 [review]. Viruses. 2020 May 26; 12(6): E582. doi: 10.3390/v12060582.

9. Lisboa Bastos M, Tavaziva G, Abidi SK, Campbell JR, Haraoui LP, Johnston JC, et al. Diagnostic accuracy of serological tests for covid-19: systematic review and meta-analysis. BMJ. 2020; 370: m2516. Published 2020 Jul 1. doi:10.1136/bmj.m2516

\section{CORRESPONDENCIA:}

Jaime Antonio Collins-Camones

jcollinslp@gmail.com

CONFLICTO DE INTERÉS: Ninguno, según los autores. FINANCIAMIENTO: Por los autores.

Fecha de recepción: 20-07-20202

Fecha de aceptación: 17-08-2020. 